\title{
3D Model Atmospheres of Red Giant Stars
}

\author{
Hans-Günter Ludwig and Matthias Steffen
}

\begin{abstract}
We provide a brief overview of the modelling of the atmospheres of red giant stars with the 3D radiation-hydrodynamics code CO5BOLD. We emphasize aspects where 3D modelling provides additional insight beyond standard hydrostatic 1D models, and comment on present modelling challenges.
\end{abstract}

\section{Introduction}

Modelling of three-dimensional (3D) atmospheres of cool stars is an active field of development (e.g. Nagendra et al., 2009), and particularly 3D models of atmospheres of red giant (RG) stars are just on the verge of becoming available for application to astrophysical problems. In an early application, Kučinskas et al. (2005) used a 3D RG model to estimate color corrections due to thermal inhomogeneities; Collet et al. (2007) considered a set of eight giant models to investigate the impact on line formation and abundances. More recently, Freytag \& Höfner (2008) developed model atmospheres of AGB stars and their winds, Dupret et al. (2009) derived the energy input to solar-like oscillations in giants from 3D models, Ramírez et al. (2010) studied convective line-shifts in the metal-poor RG HD 122563 and compared them to a 3D model, Chiavassa et al. (2011) applied global 3D models to assess effects of photometric and related astrometric variability, and Pasquini et al. (2011) took recourse to 3D dwarf and RG models to correct for convective blueshifts in high-precision, spectroscopic radial velocity measurements. While fairly exhaustive, the list of examples is still quite short, but illustrates already the variety of

H.-G. Ludwig

Zentrum für Astronomie der Universität Heidelberg, Landessternwarte, Königstuhl 12, D-69117 Heidelberg, Germany, e-mail:H.Ludwig@lsw.uni-heidelberg.de

M. Steffen Astrophysikalisches Institut Potsdam, An der Sternwarte 16, D-14482 Potsdam, Germany, e-mail: msteffendaip.de 
possible applications of 3D RG models. At the moment efforts are under way to cover the Hertzsprung-Russell diagram with 3D model atmospheres including stars in the red-giant branch (Ludwig et al., 2009a; Trampedach \& Stein, 2011).

In the following we are going to focus on aspects related to abundances from 3D models, and the theoretical calibration of the mixing-length parameter $\alpha_{\mathrm{MLT}}$ from 3D model atmospheres. Here, we are not so much presenting results as rather pointing out problems which are still lingering. We finally add some comments about predictions of the photometric micro-variability which are of interest in the context of high-precision photometry missions like CoRoT.

\section{Our model atmosphere codes}

\subsection{D model atmospheres - CO5BOLD}

Our 3D model atmospheres were calculated with the radiation-hydrodynamics code CO5BOLD (Freytag et al., 2002; Wedemeyer et al., 2004; Freytag et al., 2011). The code solves the time-dependent equations of compressible hydrodynamics coupled to radiative transfer in a constant gravity field in a Cartesian computational domain which is representative of a volume located at the stellar surface. The equation of state takes into consideration the ionization of hydrogen and helium, as well as the formation of $\mathrm{H}_{2}$ molecules according to Saha-Boltzmann statistics. Relevant thermodynamic quantities - in particular gas pressure and temperature - are tabulated as a function of gas density and internal energy. The multi-group opacities used by CO5BOLD are based on monochromatic opacities stemming from the MARCS stellar atmosphere package (Gustafsson et al., 2008) provided as function of gas pressure and temperature with high wavelength resolution. The opacities have been calculated assuming solar elemental abundances according to Grevesse \& Sauval (1998), with the exception of CNO for which values close to the recommendation of Asplund (2005) are adopted (specifically, $\mathrm{A}(\mathrm{C})=8.41, \mathrm{~A}(\mathrm{~N})=7.80, \mathrm{~A}(\mathrm{O})=8.67$ ). The metal abundances were scaled according to overall metallicity of the model assuming an enhancement of the $\alpha$-elements by +0.4 dex at metallicities $[\mathrm{M} / \mathrm{H}]<-1$.

In our RG models we typically use a number of $140 \times 140 \times 150$ to $160 \times 160 \times$ 200 points for the hydrodynamical grid. The decision about the resolution primarily hinges on the effective temperature of the model, hotter models usually require a higher resolution. The wavelength dependence of the radiation field is represented by 5 multi-group bins in the case of solar metallicity, and 6 bins at sub-solar metallicities, following the procedures laid out by Nordlund (1982); Ludwig (1992); Ludwig et al. (1994); Vögler et al. (2004). For test purposes we have calculated a few models with more bins. Since it is of relevance for the discussion later, we emphasize that all opacity sources - including scattering opacities - are treated as true absorption. The sorting into wavelength groups is done applying thresholds in logarithmic Rosseland optical depth $\{+\infty, 0.0,-1.5,-3.0,-4.5,-\infty\}$ for the 5-bin,

Page: 2 job:ludwig_roma macro:svmult.cls date/time:27-Oct-2018/18:58 
and $\{+\infty, 0.1,0.0,-1.0,-2.0,-3.0,-\infty\}$ for the 6-bin schemes. In all but one bin a switching between Rosseland and Planck averages is performed at a band-averaged Rosseland optical depth of 0.35 ; in the bin gathering the largest line opacities, the Rosseland mean opacity is used throughout. The decisions about number of bins, and sorting thresholds are motivated by comparing radiative fluxes and heating rates obtained by the binned opacities in comparison to the case of high wavelength resolution.

\section{$2.21 D$ stellar atmospheres - LHD}

Due to still present limitations in the realism (e.g. by the limited wavelength resolution) of 3D model atmospheres it is often advantageous to work differentially, and express $3 \mathrm{D}$ effects relative to a $1 \mathrm{D}$ comparison structure. To this end we developed a 1D stellar atmosphere code called LHD which employs the same opacities and equation-of-state as the 3D code CO5BOLD. The convective energy transport is modelled in the framework of mixing-length theory as described in Mihalas (1978). The resulting 1D stratifications are in hydrostatic and radiative-convective equilibrium. See Caffau et al. (2007) for more details on our approach of deriving abundance corrections.

\section{RG abundances and the issue of scattering}

Collet et al. (2007) presented 3D-1D abundance corrections for RG models at effective temperatures of around $5000 \mathrm{~K}, \log g=2.2$, and metallicities ranging from solar to $[\mathrm{M} / \mathrm{H}]=-3$, using the 3D code of Nordlund \& Stein (Stein \& Nordlund, 1998). Two similar studies were presented by Dobrovolskas et al. (2010) and Ivanauskas et al. (2010) who used CO5BOLD and LHD models at about $T_{\text {eff }}=5000 \mathrm{~K}, \log g=$ 2.5 , with metallicities down to $[\mathrm{M} / \mathrm{H}]=-3$ to derive 3D-1D abundance corrections. While the two later studies confirm the results of Collet and collaborators, showing that generally the magnitude of 3D-1D abundance corrections becomes larger towards lower metallicity, the quantitative agreement is not satisfactory. The CO5BOLD-based abundance corrections are usually noticeably smaller in magnitude, in particular at the lowest metallicities. Obviously, this is an unfortunate situation, and one would like to see a higher degree of consistency among results from different 3D codes.

In a recent paper, Collet et al. (2011) suggested the treatment of scattering in the simulations as the reason for the discrepant abundance corrections for RGs at low metallicity. The main scattering process is Rayleigh scattering by neutral hydrogen. This is perhaps the simplest case of scattering and can be modelled as coherent isotropic scattering in the continuum. Collet and collaborators implemented a proper treatment of this kind of scattering in 3D. They also put forward an approximate

Page:3 job:ludwig_roma macro:svmult.cls date/time:27-Oct-2018/18:58 
treatment of scattering by simply leaving out scattering contributions in the binned opacities in the optically thin regions. They showed that this approximate treatment provides results in close agreement with the exact treatment. They further performed a comparison with the case where scattering is treated as true absorption - as is the case in the CO5BOLD models. Their models of 2007 used the approximate treatment of scattering. The models show a sensitive dependence of the resulting temperature stratification on the treatment of scattering. In their $[M / H]=-3 R G$ model, the difference amounts to $600 \mathrm{~K}$ at optical depth $\log \tau_{\text {Ross }}=-4$ in the sense that a proper treatment of scattering leads to cooler structures in comparison to treating scattering opacities as true absorption.

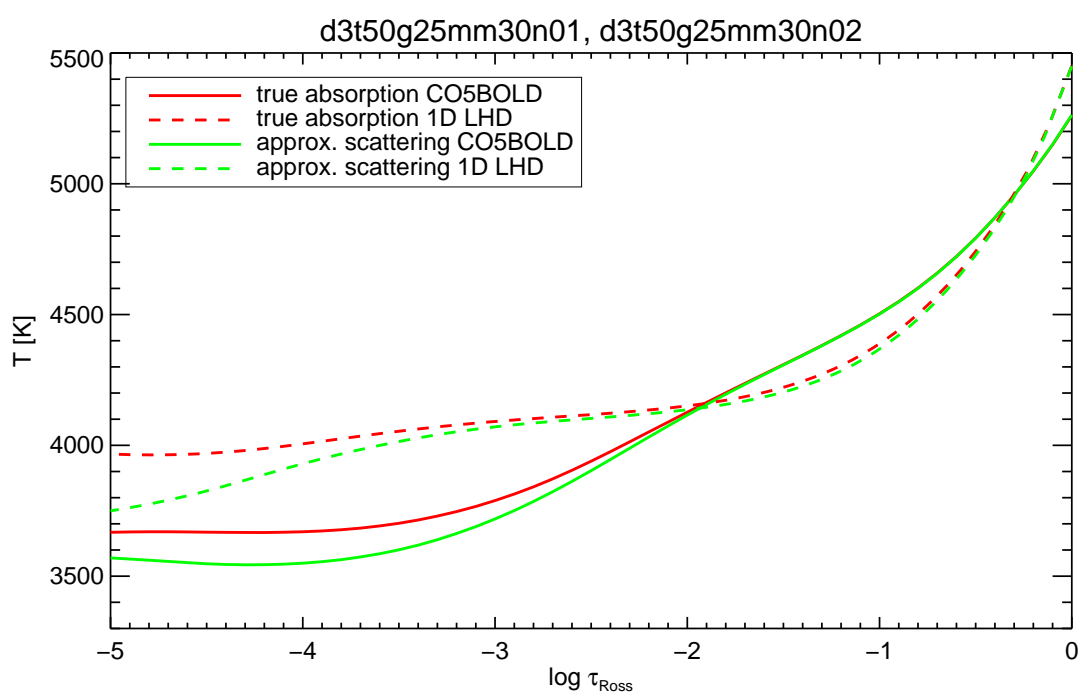

Fig. 1 Comparison of the mean temperature structures of two different 3D CO5BOLD hydrodynamical model atmospheres (solid) and associated 1D LHD models (dashed), computed with a different treatment of radiative transfer. In the first case (dark [red] curves), the continuum scattering opacity is treated as true absorption opacity, while in the second case (light [green] curves), the continuum scattering opacity is ignored in the optically thin layers. For the 3D models, averaging was performed on surfaces of constant Rosseland optical depth and over 70 equidistant snapshots covering a total of $140000 \mathrm{~s}$.

It appears plausible that the differing 3D-1D abundance corrections are a consequence of the different thermal structures resulting from the different treatment of scattering in the CO5BOLD and Nordlund-Stein class of models. To test this idea, we calculated a RG model with the same atmospheric parameters as before but with the approximate treatment of scattering as suggested by Collet et al. (2011). Figures 1 and 2 illustrate the outcome. The most striking aspect is that our models show a very much reduced sensitivity to the treatment of scattering in comparison to the models of Collet and co-workers. The approximate treatment of scattering

Page: 4 job:ludwig_roma macro:svmult.cls date/time:27-Oct-2018/18:58 


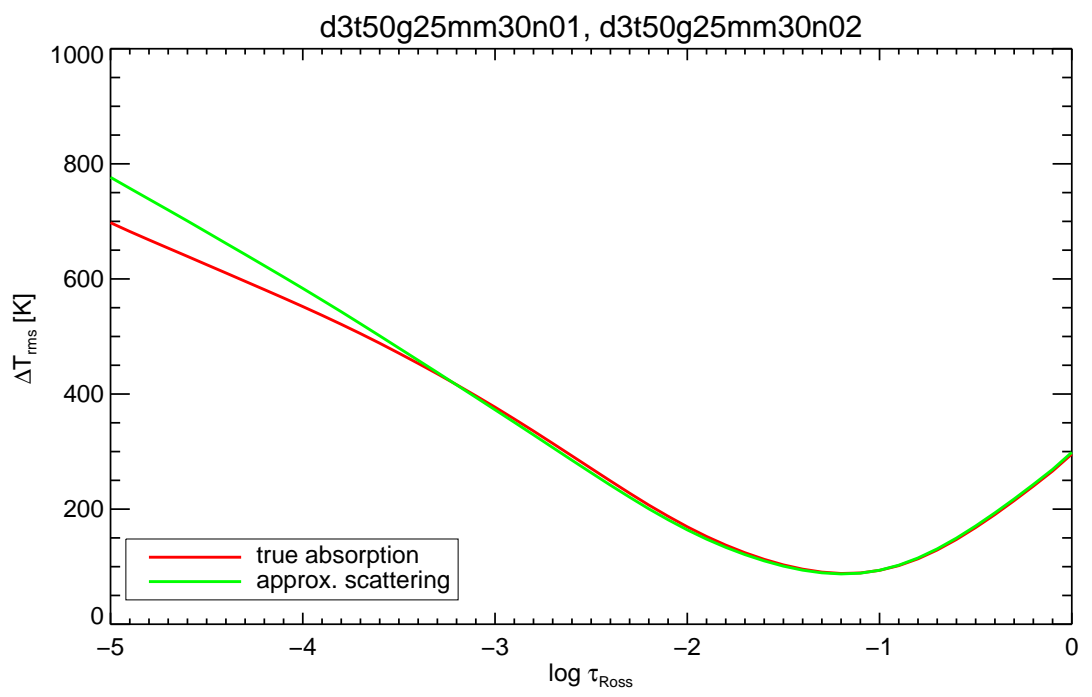

Fig. 2 Total rms temperature fluctuation $\Delta T_{\text {rms,tot }}$ as a function of Rosseland optical depth for the two 3D models shown in Fig. 1 computed as $\Delta T_{\mathrm{rms}, \mathrm{tot}}=\sqrt{\left\langle T^{2}\right\rangle_{x, y, t}-\langle T\rangle_{x, y, t}^{2}}$, where $\langle\cdot\rangle_{x, y, t}$ denotes horizontal averaging over surfaces of constant Rosseland optical depth and over time. We have verified that the amplitude of the total temperature fluctuation is completely dominated by the spatial temperature variations: $\Delta T_{\mathrm{rms}, \mathrm{tot}} \approx \Delta T_{\mathrm{rms}, \mathrm{xy}}=\left\langle\sqrt{\left\langle T^{2}\right\rangle_{x, y}-\langle T\rangle_{x, y}^{2}}\right\rangle_{t}$.

leads to a structure which is only $120 \mathrm{~K}$ cooler at $\log \tau_{\text {Ross }}=-4$, in comparison to $\approx 600 \mathrm{~K}$ found by Collet et al. (2011). This also carries over to the temperature fluctuations which are little affected by the treatment of scattering (see Fig. 2). We already emphasized the importance of a differential approach, and Fig.11also shows the effects on the associated 1D LHD models. Temperature differences between the 1D and 3D models at given optical depth are changing even less. While we did not perform spectrum synthesis calculations yet to derive new abundance corrections, we consider it as unlikely that the modest changes in the thermal structure can change our abundance corrections so much that they become consistent with the values of Collet et al. (2007).

The situation remains puzzling. The very different sensitivity to the treatment of scattering is difficult to explain. We only can hint at the differences in the calculation of the band-averaged opacities in the various codes: Collet and collaborators use intensity-averaged opacities in the optically thin regions, while we use Planckaverages - except for the band collecting the strongest lines where a Rosseland average is used throughout. We speculate that these choices, together with the definition of the opacity bins, may have a significant influence on the resulting thermal structures and their sensitivity to the treatment of scattering.

Page: 5 job:ludwig_roma macro:svmult.cls date/time:27-Oct-2018/18:58 


\section{The calibration of $\alpha_{\mathrm{MLT}}$ and turbulent pressure}

It is well known from the theory of stellar structure that convection is generally an efficient means of transporting energy, and that it establishes a thermal structure close to adiabatic. Only in the vicinity of the boundaries of convective regions noticeable deviations from adiabaticity occur. In convective envelopes of late-type stars the upper boundary of the convective envelope - usually located close to or even in the optically thin layers - constitutes the bottle-neck for the energy transport through the stellar envelope assigning a special role to it. Despite its small geometrical extent and low mass, it largely determines the properties of the convective envelope as a whole. It is the value of the entropy of the adiabatically stratified bulk of the convective $s_{\text {env }}$ which is most important from the point of view of stellar structure since it influences the resulting radius and effective temperature of a stellar model. $s_{\text {env }}$ is controlled by the efficiency of convective and radiative energy transport in the thin, superadiabatically stratified surface layers. 3D model atmospheres can be applied to model this region, and allow to quantify the mutual efficiency of the convective and radiative energy transport, and to predict $s_{\mathrm{env}}$. Comparing the model predictions to standard 1D models based on mixing-length theory (MLT) the value of $s_{\text {env }}$ can be translated into a corresponding mixing-length parameter $\alpha_{\text {MLT }}$ (Trampedach et al., 1999; Ludwig et al., 1999, 2008).

In stellar evolution calculations the free mixing-length parameter is usually calibrated against the Sun. However, it is unclear whether mixing-length theory provides a suitable scaling of the convective efficiency at constant $\alpha_{\text {MLT }}$ across the Hertzsprung-Russell diagram. The depth of the surface convective envelope and the related $\alpha_{\mathrm{MLT}}$ can be constraint by asteroseismology. However, degeneracies with other parameters often make it difficult to obtain a unique solution (e.g. Goupil et al., 2011). Hence, it would be useful to have an independent estimate available which $3 \mathrm{D}$ models can provide in principle.

In main-sequence models turbulent pressure plays generally only a minor role but becomes relatively more important towards lower gravities - and causes trouble when one is interested in a well-defined calibration of the mixing-length parameter. Figure 3 shows the average temperature profile of a 3D red giant model ( $T_{\text {eff }} \approx 3600 \mathrm{~K}, \log g=1.0,[\mathrm{M} / \mathrm{H}]=0.0$ ) in comparison to standard 1D model atmospheres of the same atmospheric parameters. While the turbulent pressure $P_{\text {turb }}$ is naturally included in the 3D models, it is modelled in a ad-hoc fashion in 1D models, assuming a parameterisation $P_{\text {turb }}=f_{\text {turb }} \rho v_{\mathrm{c}}^{2}$, where $f_{\text {turb }}$ is a free parameter of order unity, $\rho$ the mass density and $v_{\mathrm{c}}$ the convective velocity according MLT.

Figure 3 shows that it is essentially impossible to reproduce the mean thermal profile of the 3D model with any of the 1D models - irrespective of the choice of $\alpha_{\text {MLT }}$ and $f_{\text {turb }}$. The failure is related to the local nature of MLT confining the action of the turbulent pressure gradients strictly to the convectively unstable regions. While formally one can still match $\mathrm{s}_{\mathrm{env}}$ of the 3D model by a 1D profile with suitably chosen $\alpha_{\text {MLT }}$ and/or $f_{\text {turb }}$ such a match becomes physically little motivated, and is unlikely to provide a robust scaling with changing atmospheric parameters. An improved 1D convection description including non-local effects like

Page: 6 job:ludwig_roma macro:svmult.cls date/time:27-Oct-2018/18:58 


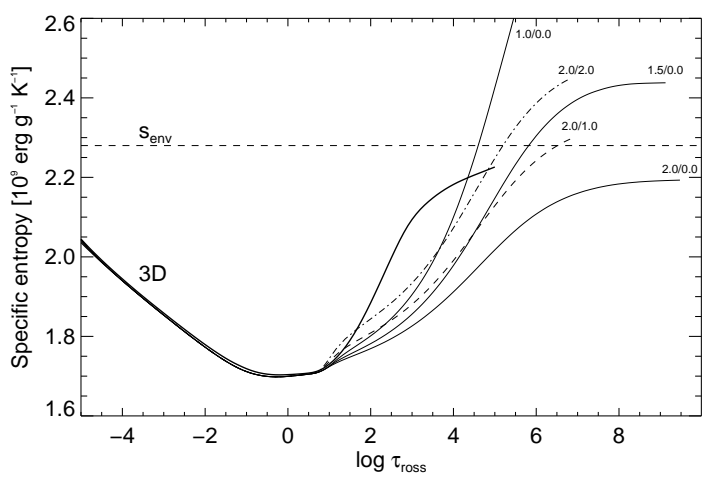

Fig. 3 Entropy-optical depth profiles (horizontal and temporal average) of a 3D red giant model (thick solid line) in comparison to 1D stellar atmosphere models of different $\alpha_{\mathrm{MLT}}$ leaving out (thin solid lines) or including (dashed and dashed-dotted lines) turbulent pressure. The lines are labeled with values $\alpha_{\mathrm{MLT}} / f_{\text {turb }}$ (details see text). The horizontal dashed line indicates the value of $\mathrm{s}_{\mathrm{env}}$ as predicted by the 3D model.

overshooting is clearly desirable to handle this situation. Empirical calibrations of $\alpha_{\text {MLT }}$ using giants are likely to suffer from ambiguities related to the way turbulent pressure is treated in the 1D models. One may take the result as an indication that taking recourse to $1 \mathrm{D}$ models is not warranted, and one may give up the benefits of a differential approach by relating 3D to 1D structures. Alternatively, one may take the absolute entropy of the convective envelope (perhaps translated to equivalent pressure-temperature pairs) as predicted by the $3 \mathrm{D}$ model as constraint to be matched in 1D stellar structure models.

\section{Granulation-related photometric micro-variability}

High-precision photometry of satellite missions (foremost Most, CoRoT, Kepler) allow the detection of stellar variability associated with the random changes of the granulation pattern on the surfaces of late-tape stars - by asteroseismologists usually referred to as "granular background noise". 3D model atmospheres represent the granulation pattern in detail and allow to predict the power spectrum of the variability signal (Trampedach et al., 1998; Svensson \& Ludwig, 2005; Ludwig, 2006). Despite this possibility, no comprehensive theoretical study has been conducted so far. One of the reasons is that long time series need to be calculated to collect sufficient statistics, which is computationally demanding. The F-dwarf HD 49933 - a prominent CoRoT-target - is an exception for which Ludwig et al. (2009b) performed an analysis. However, the growing body of observational data in particular for giant stars should motivate further efforts in this direction. Recently, Kjeldsen \& Bedding (2011) suggested a new scaling relation for the amplitude of solar-like oscillations, and also discuss the scaling of the granulation background signal. It would be in-

Page: 7 job:ludwig_roma macro:svmult.cls date/time:27-Oct-2018/18:58 
teresting to see whether 3D model atmospheres can lend further support to the suggested relations.

To illustrate the feasibility, we show in Fig. 4 a rough comparison of the photometric variability between the RG HD 181907 (HR 7349) and predictions from two 3D models. The plot focuses on the frequency region where the granulation-related signal is expected. CoRoT acquired a high-quality time series for HD 181907; Carrier et al. (2010) give atmospheric parameters $4780 \pm 80 / 2.78 \pm 0.16 /-0.08 \pm$ $0.10\left(T_{\text {eff }} / \log g /[\mathrm{M} / \mathrm{H}]\right)$. The two 3D models have atmospheric parameters $4500 / 2.5 / 0.0$ and 5000/2.5/0.0, bracketing the star in effective temperature, as well as having comparable surface gravity and metallicity. Although no dedicated modelling was performed, the spectra appear quite similar.

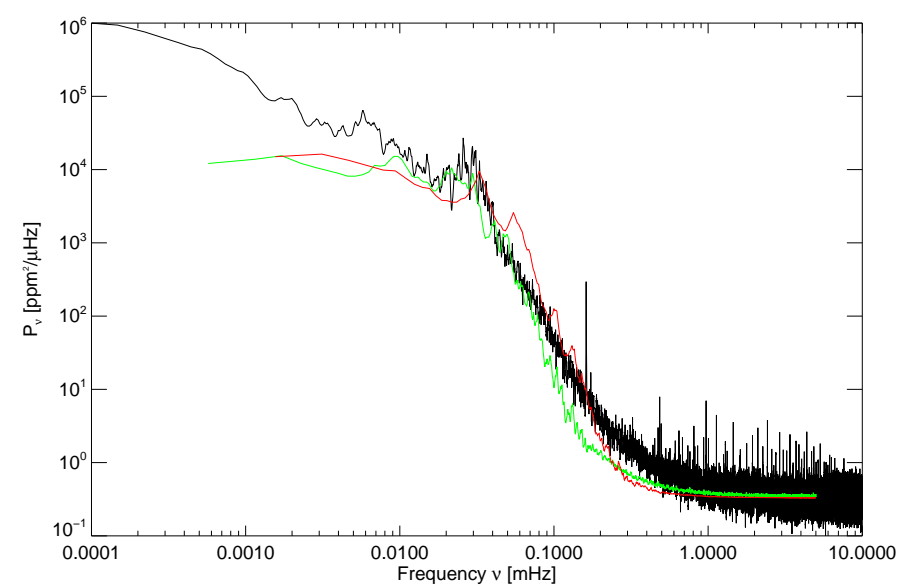

Fig. 4 Comparison of power spectra of photometric variability. Black line: HD 181907 as observed by CoRoT. The predicted power spectra from two 3D models are depicted with light [green ] $\left(T_{\text {eff }}=4500 \mathrm{~K}\right)$ and dark grey $[\mathrm{red}]\left(T_{\text {eff }}=5000 \mathrm{~K}\right)$ lines. Further details see text.

\section{Concluding remarks}

3D model atmospheres of cool stars, including red giants, have reached a level of realism which allows a direct confrontation with observations. In some areas they allow to make predictions beyond the capabilities of classical 1D models. However, as we have seen there still exist modelling challenges, and last but not least quite some work is still necessary to fully exploit the potential of such models.

Page: 8 job:ludwig_roma macro:svmult.cls date/time:27-0ct-2018/18:58 


\section{References}

Asplund, M. 2005, ARA\&A, 43, 481

Caffau, E., Faraggiana, R., Bonifacio, P., Ludwig, H.-G., \& Steffen, M. 2007, A\&A, 470, 699

Carrier, F., Morel, T., Miglio, A., et al. 2010, Ap\&SS , 328, 83

Chiavassa, A., Pasquato, E., Jorissen, A., et al. 2011, A\&A, 528, A120

Collet, R., Asplund, M., \& Trampedach, R. 2007, A\&A, 469, 687

Collet, R., Hayek, W., Asplund, M., et al. 2011, A\&A, 528, A32

Dobrovolskas, V., Kučinskas, A., Ludwig, H.-G., et al. 2010, ArXiv e-prints

Dupret, M., Belkacem, K., Samadi, R., et al. 2009, A\&A, 506, 57

Freytag, B. \& Höfner, S. 2008, A\&A, 483, 571

Freytag, B., Steffen, M., \& Dorch, B. 2002, Astronomische Nachrichten, 323, 213

Freytag, B., Steffen, M., Ludwig, H.-G., et al. 2011, J.Comp.Phys., submitted

Goupil, M. J., Lebreton, Y., Marques, J. P., et al. 2011, Journal of Physics Conference Series, 271, 012032

Grevesse, N. \& Sauval, A. J. 1998, Space Science Rev. , 85, 161

Gustafsson, B., Edvardsson, B., Eriksson, K., et al. 2008, A\&A, 486, 951

Ivanauskas, A., Kucinskas, A., Ludwig, H. G., \& Caffau, E. 2010, in Nuclei in the Cosmos.

Kjeldsen, H. \& Bedding, T. R. 2011, A\&A, 529, L8

Kučinskas, A., Hauschildt, P. H., Ludwig, H.-G., et al. 2005, A\&A, 442, 281

Ludwig, H.-G. 1992, PhD thesis, University of Kiel

Ludwig, H.-G. 2006, A\&A, 445, 661

Ludwig, H.-G., Caffau, E., \& Kučinskas, A. 2008, in IAU Symposium, Vol. 252, IAU Symposium, ed. L. Deng \& K. L. Chan, 75-81

Ludwig, H.-G., Caffau, E., Steffen, M., et al. 2009a, MmSAI, 80, 711

Ludwig, H.-G., Freytag, B., \& Steffen, M. 1999, A\&A, 346, 111

Ludwig, H.-G., Jordan, S., \& Steffen, M. 1994, A\&A, 284, 105

Ludwig, H.-G., Samadi, R., Steffen, M., et al. 2009b, A\&A, 506, 167

Mihalas, D. 1978, Stellar atmospheres, 2nd edition

Nagendra, K. N., Bonifacio, P., \& Ludwig, H.-G. 2009, MmSAI, 80, 601

Nordlund, A. 1982, A\&A, 107, 1

Pasquini, L., Melo, C., Chavero, C., et al. 2011, A\&A, 526, A127

Ramírez, I., Collet, R., Lambert, D. L., Allende Prieto, C., \& Asplund, M. 2010, ApJ , 725, L223

Stein, R. F. \& Nordlund, A. 1998, ApJ , 499, 914

Svensson, F. \& Ludwig, H.-G. 2005, in ESA Special Publication, Vol. 560, 13th Cambridge Workshop on Cool Stars, Stellar Systems and the Sun, ed. F. Favata, G. A. J. Hussain, \& B. Battrick, 979

Trampedach, R., Christensen-Dalsgaard, J., Nordlund, A., \& Stein, R. F. 1998, in The First MONS Workshop: Science with a Small Space Telescope, ed. H. Kjeldsen \& T. R. Bedding, 59

Trampedach, R. \& Stein, R. F. 2011, ApJ , 731, 78

Trampedach, R., Stein, R. F., Christensen-Dalsgaard, J., \& Nordlund, Å. 1999, in Astronomical Society of the Pacific Conference Series, Vol. 173, Stellar Structure: Theory and Test of Connective Energy Transport, ed. A. Gimenez, E. F. Guinan, \& B. Montesinos, 233

Vögler, A., Bruls, J. H. M. J., \& Schüssler, M. 2004, A\&A, 421, 741

Wedemeyer, S., Freytag, B., Steffen, M., Ludwig, H.-G., \& Holweger, H. 2004, A\&A, 414, 1121 